\title{
What determines glomerular capillary permeability?
}

\author{
William M. Deen
}

Department of Chemical Engineering and Division of Biological Engineering, Massachusetts Institute of Technology, Cambridge, Massachusetts, USA.

\begin{abstract}
There have been exciting recent advances in our understanding of the structural and molecular biology of the glomerular slit diaphragm, as described in a report in this issue of the JCI (see the related article beginning on page 1475). These findings, combined with data on the permeability of the basement membrane and evidence that the endothelium may be a more important barrier than often supposed, are allowing a clearer understanding to emerge of how the 3 parts of the glomerular capillary wall jointly determine its functional properties.
\end{abstract}

The glomerular capillary wall is a living ultrafiltration membrane. It permits water and small solutes to pass readily into Bowman's space, while normally rejecting albumin and other large proteins with great efficiency. As shown in Figure 1, the glomerular capillary wall consists of a fenestrated endothelium, the glomerular basement membrane (GBM), and the interdigitated foot processes of epithelial cells (podocytes). The filtration pathway is extracellular; that is, water and filtered solutes pass through the fenestrae, across the GBM, and through filtration slits bounded by the foot processes. The filtration slits are spanned by porous slit diaphragms.

The strategic location of the slit diaphragm has long suggested that it might play a crucial role in restricting the passage of solutes on the basis of molecular size. Thus, disruption of slit diaphragms might underlie the proteinuria that is a hallmark of kidney disease. The most influential study of the fine structure of the slit diaphragm has been that of Rodewald and Karnovsky (1). They interpreted EM images of the slit diaphragm as showing a zipperlike structure in which a central fiber, in a plane parallel to that of the podocyte membranes, is connected to those membranes by perpendicular bridge fibers. Viewed in the direction of filtrate flow, as shown in Figure 2 , the proposed structure exhibits rectangular openings with dimensions of $4 \times 14 \mathrm{~nm}$,

Nonstandard abbreviations used: GBM, glomerular basement membrane; $\Theta$, sieving coefficient; $\Theta_{b m}$, basement membrane sieving coefficient; $\Theta_{e n}$, endothelium sieving coefficient; $\Theta_{e p}$, epithelium sieving coefficient.

Conflict of interest: The author has declared that no conflict of interest exists.

Citation for this article: J. Clin. Invest. 114:1412-1414 (2004). doi:10.1172/JCI200423577. alternating from side to side. Those openings correspond almost exactly to the molecular dimensions calculated from the hydrodynamic properties of albumin, when it is modeled as a prolate spheroid (2). That, and its elegant regularity, have made the zipper structure very appealing. However, the dimensions of the openings shown in Figure 2 suggest that spherical molecules of even 2-nm radius will be excluded from Bowman's space, whereas such molecules actually exhibit little restriction (3).
Figure 1 Figure is not drawn to scale.

\section{New findings concerning the slit diaphragm}

In this issue of the JCI, the study by Wartiovaara et al. (4) advances the understanding of the structure and composition of the slit diaphragm in 2 important ways. First, the authors' novel application of electron tomography provides a more detailed view of the porous structure than was heretofore possible. Certain aspects of the classical zipper structure are supported (e.g., a central dense region with roughly albumin molecule-sized openings on either side), but the pores appear to be more tortuous and irregular than previously supposed. A second advance concerns the identity of the macromolecules that constitute the slit diaphragm. New evidence is provided for the crucial role of nephrin, a transmembrane protein that is expressed by podocytes and localized in the slit diaphragm area, in

\section{Bowman's space}

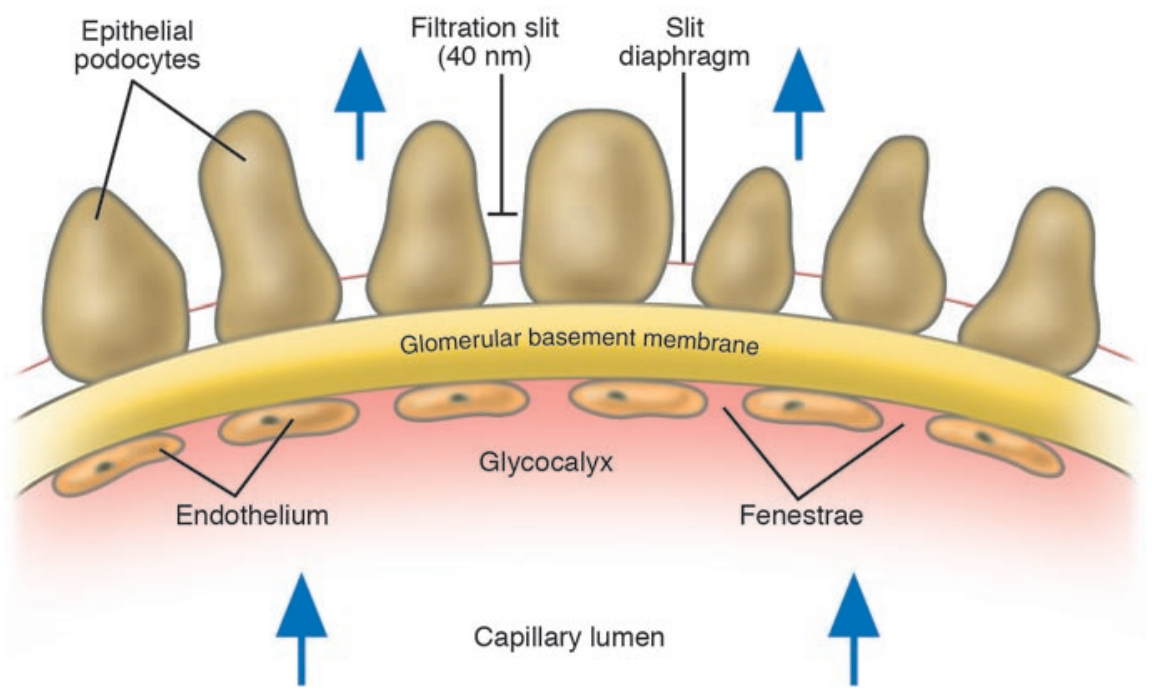

Glomerular capillary wall, consisting principally of a fenestrated endothelium, a basement membrane, and epithelial foot processes. The foot processes form filtration slits spanned by slit diaphragms. Also shown is the endothelial cell coat, or glycocalyx. Some approximate dimensions are $(8,10)$ : minimum diameter of fenestra, $30 \mathrm{~nm}$; GBM thickness, 200-400 nm (depending on species); width of filtration slit, $40 \mathrm{~nm}$. The glycocalyx thickness is uncertain. 


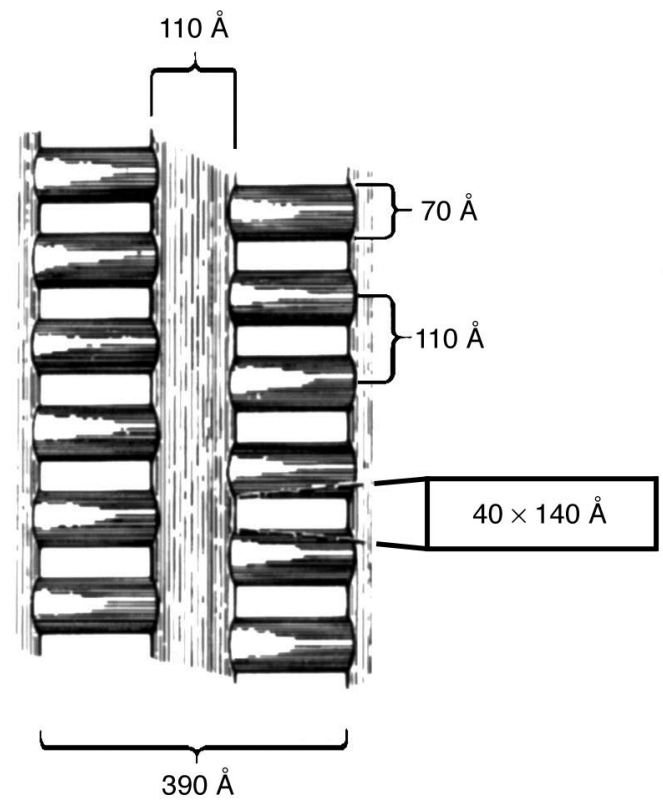

Figure 2

Slit diaphragm structure proposed by Rodewald and Karnovsky (1). A central filament is connected to the podocyte membranes by alternating bridge fibers. The view is in the direction of filtrate flow. Figure reprinted with permission from the Proceedings of the National Academy of Sciences (17).

forming this structure. Indeed, the fibers that constitute the slit diaphragm appear to be formed largely by the association of extracellular strands of nephrin. When nephrin is abnormal or absent, as is the case in individuals with Finnish congenital nephrotic syndrome (which is caused by a mutation in the nephrin gene) or in nephrin-knockout mice, ordered slit diaphragm structures are no longer evident, and proteinuria results. That disruption of slit diaphragms is sufficient to cause proteinuria seems indisputable.

\section{Effects of other structures on glomerular permeability}

How does the rest of the glomerular capillary wall influence its permeability? A measure of the overall selectivity of the glomerular barrier is the sieving coefficient $(\Theta)$, which is the concentration of a given molecule in Bowman's space divided by that in plasma. For a freely filtered solute, $\Theta=1$; for a completely rejected solute, $\Theta=0$. The overall sieving coefficient is the product of the sieving coefficients of the individual layers; $\Theta=\Theta_{e n} \Theta_{b m} \Theta_{e p}$, where $\Theta_{e n}, \Theta_{b m}$, and $\Theta_{e p}$ are the downstream-toupstream concentration ratios for the endothelium, basement membrane, and epithelium (slit diaphragm), respectively.
Thus, in principle, a large increase in $\Theta$ for albumin, and consequent albuminuria, might result from increases in $\Theta_{e n}$ or $\Theta_{b m}$ and not just $\Theta_{e p}$.

The ability to isolate GBM from rat glomeruli and to perform filtration and diffusion experiments in vitro has allowed the transport properties of the GBM to be quantified $(5-7)$. As has been reviewed (8), the GBM is a very size-selective structure. For example, the diffusional permeability of GBM to an albumin-size molecule is less than $1 \%$ that of a layer of water of equal thickness. Nonetheless, given its measured transport properties, the linear dimensions and flow rates in vivo, and the placement of the GBM just upstream from a moderately to highly selective barrier (the slit diaphragm), the size selectivity of the GBM is expected to be almost completely masked. In other words, for the intact capillary wall in vivo, it is calculated that $\Theta_{b m} \cong 1$ (8). Consistent with this prediction is that deletion of heparan sulfate, a major structural component of the GBM, does not lead to proteinuria (9). It has been estimated that the GBM, while having little effect on macromolecular sieving in vivo, accounts for $50-70 \%$ of the resistance to filtrate flow $(8,10)$. Thus, the GBM is the single most important determinant of the glomerular hydraulic permeability.
The endothelium has often been viewed as an insignificant part of the barrier, but this interpretation seems unjustified. That $\Theta_{e n} \ll 1$ for albumin is suggested by tracer visualization studies (11) and by the proteinuria that accompanies preeclampsia, which selectively affects the endothelium (12). The fenestrae themselves are too large to effectively sieve macromolecules, which suggests that the barrier properties of the endothelium are derived from the cell coat, or glycocalyx, that fills the fenestrae and extends into the capillary lumen. Special fixation techniques have permitted the glomerular endothelial glycocalyx to be visualized by EM (13), and similar cell coats have been shown to be functionally significant in extrarenal capillaries (14). Evidence that the endothelial glycocalyx may play a role in glomerular size and charge selectivity has been reviewed recently (15).

\section{Potential origins of proteinuria}

Simple calculations suggest that proteinuria need not have a single origin. Assume that $\Theta=4 \times 10^{-4}$ for albumin in healthy humans, which is similar to what has been determined by micropuncture in rats (16). Consider a hypothetical situation in which the endothelium and epithelium are equally selective, so that $\Theta=\Theta_{e n} \Theta_{b m} \Theta_{e p}=$ $\left(2 \times 10^{-2}\right)(1)\left(2 \times 10^{-2}\right)=4 \times 10^{-4}$. With a GFR of $180 \mathrm{l}$ /day and a plasma albumin concentration of $50 \mathrm{~g} / 1$, the filtered load would be $(180 \mathrm{l} /$ day $)(50 \mathrm{~g} / \mathrm{l})\left(4 \times 10^{-4}\right)=3.6 \mathrm{~g} /$ day. The normal absence of albuminuria indicates that the renal tubules can reabsorb such an amount. Now, if the endothelial barrier were removed (i.e., $\Theta_{e n}=1$ ) and nothing else altered, $\Theta$ would increase to $2 \times 10^{-2}$ and the filtered load would rise to $180 \mathrm{~g} /$ day, likely overwhelming the tubule reabsorptive process. If only the slit diaphragms were removed (i.e., $\Theta_{e p}=1$ ), the filtered load would increase to a smaller, although still massive, level of $72 \mathrm{~g} /$ day; the difference is that $\Theta_{b m}$ is predicted to decline in this case to about 0.4 (8). Of course, the normal $\Theta_{e n}$ and $\Theta_{\text {ep }}$ may not be equal, and the albumin reabsorptive capacity is not known with precision. However, even if $\Theta_{e n}$ and $\Theta_{e p}$ differ by as much as 100 -fold in the normal glomerulus (e.g., with one as large as 0.1 and the other as small as $10^{-3}$ ), clinical albuminuria could easily result from either endothelial or epithelial defects. Thus, in considering the determinants of glomerular capillary permeability, one should avoid the temptation to focus exclusively on any one of the 3 layers, instead keeping in mind that the 3 act in concert. 
Address correspondence to: William M. Deen, Department of Chemical Engineering and Division of Biological Engineering, Massachusetts Institute of Technology, 77 Massachusetts Avenue, Room 66-572, Cambridge, Massachusetts 02139, USA. Phone: (617) 253-4535; Fax: (617) 253-2072; E-mail:wmdeen@mit.edu.

1. Rodewald, R., and Karnovsky, M.J. 1974. Porous substructure of the glomerular slit diaphragm in the rat and mouse. J. Cell Biol. 60:423-433.

2. Lazzara, M.J., and Deen, W.M. 2004. Effects of concentration on the partitioning of macromolecule mixtures in agarose gels. J. Colloid Interface Sci. 272:288-297.

3. Blouch, K., et al. 1997. Molecular configuration and glomerular size selectivity in healthy and nephrotic humans. Am. J. Physiol. 273:F430-F437.

4. Wartiovaara, J., et al. 2004. Nephrin strands contribute to a porous slit diaphragm scaffold as revealed by electron tomography. J. Clin. Invest.
114:1475-1483. doi:10.1172/JCI200422562.

5. Daniels, B.S., Hauser, E.B., Deen, W.M., and Hostetter, T.H. 1992. Glomerular basement membrane: in vitro studies of water and protein permeability. Am. J. Physiol. 262:F919-F926.

6. Edwards, A., Deen, W.M., and Daniels, B.S. 1997. Hindered transport of macromolecules in isolated glomeruli. I. Diffusion across intact and cell-free capillaries. Biophys. J. 72:204-213.

7. Bolton, G.R., Deen, W.M., and Daniels, B.S. 1998 Assessment of the charge-selectivity of glomerular basement membrane using ficoll sulfate. Am. J. Physiol. 274:F889-F896.

8. Deen, W.M., Lazzara, M.J., and Myers, B.D. 2001. Structural determinants of glomerular permeability. Am. J. Physiol. 281:F579-F596.

9. Rossi, M., et al. 2003. Heparan sulfate chains of perlecan are indispensable in the lens capsule but not in the kidney. EMBOJ. 22:236-245.

10. Drumond, M.C., and Deen, W.M. 1994. Structural determinants of glomerular hydraulic permeability. Am. J. Physiol. 26:F1-F12.

11. Ryan, G.B., and Karnovsky, M.J. 1976. Distribution of endogenous albumin in the rat glomerulus:
Role of hemodynamic factors in glomerular barrier function. Kidney Int. 9:36-45.

12. Maynard, S.E., et al. 2003. Excess placental soluble fms-like tyrosine kinase 1 (sFlt1) may contribute to endothelial dysfunction, hypertension, and proteinuria in preeclampsia. J. Clin. Invest. 111:649-658. doi:10.1172/JCI200317189.

13. Rostgaard, I., and Qvortrup, K. 1997. Electron microscopic demonstration of filamentous molecular sieve plugs in capillary fenestrae. Microvasc. Res. 53:1-13.

14. Henry, C.B., and Duling, B.R. 1999. Permeation of the luminal capillary glycocalyx is determined by hyaluronan. Am. J. Physiol. 277:H508-H514.

15. Haraldsson, B., and Sorensson, J. 2004. Why do we not all have proteinuria? An update of our current understanding of the glomerular barrier. News Physiol. Sci. 19:7-10

16. Tojo, A., and Endou, H. 1992. Intrarenal handling of proteins in rats using fractional micropuncture technique. Am. J. Physiol. 263:F601-F606.

17. Ruotsalainen, V., et al. 1999. Nephrin is specifically located at the slit diaphragm of glomerular podocytes. Proc. Natl. Acad. Sci. U. S. A. 96:7962-7967.

\title{
Genes and pathophysiology of type 2 diabetes: more than just the Randle cycle all over again
}

\author{
Alan R. Shuldiner and John C. McLenithan \\ Division of Endocrinology, Diabetes and Nutrition, University of Maryland School of Medicine, Baltimore, Maryland, USA. \\ Geriatric Research and Education Clinical Center, Veterans Administration Medical Center, Baltimore, Maryland, USA.
}

\begin{abstract}
The Randle cycle, which has been invoked to explain the reciprocal relationship between fatty acid oxidation and glucose oxidation, has long been implicated as a potential mechanism for hyperglycemia and type 2 diabetes mellitus (T2DM). Now genetic, functional genomic, and transgenic approaches have identified PPAR $\gamma$ coactivators (PGC-1 $\alpha$ and PGC-1 $\beta$ ) as key regulators of mitochondrial number and function. They regulate adaptive thermogenesis as well as glucose and fat oxidation in muscle and fat tissue, gluconeogenesis in liver, and even glucose-regulated insulin secretion in $\beta$ cells. PGC-1 $\alpha$ and PGC-1 $\beta$ mRNA levels and the mitochondrial genes they regulate are decreased in muscle of people with prediabetes and T2DM. A new report indicates that PGC-1 $\alpha$ and PGC-1 $\beta$ mRNA levels decrease with age in individuals with a genetic variant in PGC-1 $\alpha$, and these decreases correlate with alterations in whole-body glucose and fatty acid oxidation (see the related article beginning on page 1518). These findings provide insights into how aging modifies genetic susceptibility to alterations in oxidative phosphorylation and T2DM.
\end{abstract}

Type 2 diabetes mellitus (T2DM), considered a rare disease no more than 100 years ago, is now an epidemic in the United States

Nonstandard abbreviations used: HNF, hepatocyte nuclear factor; IPF-1, insulin promoter factor-1; MIDD, maternally inherited diabetes and deafness; OXPHOS, oxidative phosphorylation; PGC, PPAR $\gamma$ coactivator; T2DM, type 2 diabetes mellitus; $\mathrm{VO}_{2 \max }$, total body aerobic capacity.

Conflict of interest: Alan R. Shuldiner serves as a consultant for Amgen, Pfizer, Inc., and Serono, Inc. He receives research support from Merck \& Co., Inc.

Citation for this article: J. Clin. Invest. 114:1414-1417 (2004). doi:10.1172/JCI200423586. and other industrialized countries. Obesity and advancing age are potent risk factors for T2DM, pointing to lifestyle changes of the 20th century that are responsible for the current epidemic. However, despite our diabetogenic environment, some individuals develop diabetes and others do not. Multiple studies provide evidence that genetic factors are important contributors to the large inter-individual variation in diabetes susceptibility $(1,2)$. Identification of T2DM susceptibility genes has proven challenging, in part due to the heteroge- neous and polygenic nature of the condition and due to our limited understanding of its underlying pathophysiology. In the past decade, new and powerful tools for probing the molecular, genetic, and pathophysiological basis of glucose and energy homeostasis have provided key insights into the molecular basis of diabetes. Some of these insights have proven quite surprising based upon the current state of knowledge, while others have been logical extensions of the state of the field.

\section{Genetics of diabetes: what we do know}

Simply put, diabetes occurs as a result of an absolute or relative deficiency of insulin. The former occurs in autoimmune forms of diabetes, e.g., type 1 diabetes mellitus, or latent autoimmune diabetes in adults, in which progressive destruction of insulin-secreting $\beta$ cells leads to an absolute deficiency of insulin. Relative insulin deficiency is far more pervasive and in its most common form, T2DM, is caused by insulin resistance (most often due to obesity) coupled with progressive failure of the $\beta$ cell to secrete sufficient insulin to compensate for the increased insulin resis- 